(150-160bpm), mean blood pressure $(43-46 \mathrm{mmHg})$ and RI during infusion. Plasma lactate concentrations decreased and urine output improved over $48 \mathrm{~h}$. One patient developed stroke pre-treatment; another had persistent hypotension after milrinone. Sixteen (94\%) neonates survived to hospital discharge.

Conclusions Milrinone improves CO and cerebral blood Vm without significant effects on blood pressure and RI. (Funded by Stollery Children's Hospital Foundation)

\section{THE REGIONAL HEMODYNAMIC EFFECTS OF DOXYCYCLINE IN NEWBORN PIGLETS WITH ASPHYXIA-REOXYGENATION}

doi:10.1136/archdischild-2012-302724.0320

IJ LaBossiere, 'JS Pelletier, 'D Bigam, 1,2PY Cheung. 'Surgery; ${ }^{2}$ Pediatrics and Pharmacology, University of Alberta, Edmonton, AB, Canada

Background Neonatal asphyxia has significant morbidities including hypoxic-ischemic encephalopathy, necrotizing enterocolitis and acute renal failure. These complications are associated with regional perfusion deficits. During reoxygenation, oxygen free radicals are produced and can activate matrix metalloproteinase-2, leading to cardiovascular dysfunction. There is little information regarding the effect of doxycycline, a known inhibitor of matrix metalloproteinase -2 , on regional perfusion. We hypothesized that doxycycline would improve regional hemodynamics during recovery in asphyxiated newborn piglets.

Methods Piglets (1-5 days old) were acutely instrumented for continuous monitoring of blood flow at the left common carotid, superior mesenteric and renal arteries (CAFI, SMAFI and RAFI, respectively). After stabilization, $2 \mathrm{hrs}$ of normocapnic alveolar hypoxia (10-15\% oxygen) was induced followed by 4 hrs of reoxygenation (21\% oxygen). Piglets were blindly randomized to receive either normal saline or doxycycline $(3,10$, or $30 \mathrm{mg} / \mathrm{kg}$ ) intravenously 5 minutes into reoxygenation ( $n=7 /$ group). Sham-operated piglets $(\mathrm{n}=5)$ received no hypoxia-reoxygenation.

Results All piglets had regional perfusion deficits at $2 \mathrm{hrs}$ of hypoxia (CAFI: $76 \pm[S D] 16 \%$; SMAFI: $57 \pm 21 \%$; RAFI: $11 \pm 10 \%$ of respective normoxic baseline). During 4 hrs of reoxygenation, doxycycline at $30 \mathrm{mg} / \mathrm{kg}$ increased RAFI $(\mathrm{p}<0.001)$ and CAFI $(\mathrm{p}=0.06$ ) (at 240min reoxygenation: $66 \pm 33 \%$ vs. $36 \pm 21 \% \quad[p=0.04]$ and $92 \pm 52 \%$ vs. $66 \pm 22 \%$ [p=0.09] for controls, respectively). Despite attenuating mesenteric hyperemia at $10 \mathrm{~min}$ of reoxygenation $(\mathrm{p}=0.03)$, there was no difference in SMAFI at the end of reoxygenation.

Conclusions In newborn piglets with hypoxia-reoxygenation, post-resuscitation administration of high-dose intravenous doxycycline improves carotid and renal hemodynamics and may attenuate transient mesenteric hyperemia during recovery.

Funded by Canadian Institutes of Health Research (CIHR)

\section{EARLY TARGETED CLOSURE OF PATENT DUCTUS ARTERIOSUS IN EXTREME PRETERM BABIES REDUCES BRONCHOPULMONARY DYSPLASIA}

doi:10.1136/archdischild-2012-302724.0321

'P Mallya, 'J Sundaram, 1,2S Gupta. 'Paediatrics and Neonatal Medicine, University Hospital of North Tees, Stockton; ${ }^{2}$ University of Durham, Durham, UK

Background PDA is common among very low birth weight babies. There is however growing controversy regarding treatment and clinicians are uncertain to treat or not to treat a PDA.

Objective To compare mortality and complications of prematurity in the following groups

1. Effect of gestation; babies $\leq 28$ weeks $\& 29-31$ weeks

2. Early $(<72 \mathrm{hrs})$ treatment vs. later symptomatic treatment of a PDA

3. Treatment vs. no treatment of a significant PDA
Methods All babies born < 32 weeks gestation were included in this study. A total of 223 babies met the inclusion criteria from January 2009 to September 2011. Of these 22 were excluded from analysis due to incomplete data. SPSS version 17(C) was used for data analysis.

Results

1. Babies $\leq 28$ weeks compared to 29-31weeks gestation had significantly higher PDA (61\%vs.23\%; p<0.05), Bronchopulmonary dysplasia (BPD) (40\%vs.12\%; $\mathrm{p}<0.05)$, duration of respiratory support (27days vs. 9 days; $p<0.05$ ) and mortality (16\%vs. $0 \%$; $<<0.05)$

2. Among babies with significant PDA, BPD was significantly reduced with early treatment of a PDA as compared to later symptomatic treatment (66.7\%vs.82.3\%; $\mathrm{p}<0.05$ )

3. There was significantly higher incidence of death among babies not treated for PDA as compared to those who received treatment ( $29 \%$ vs. $0 \%$; $p<0.001)$

\section{Conclusion}

1. Babies born $\leq 28$ weeks have higher burden of PDA, mortality and complications of prematurity.

2. Treatment of PDA significantly reduces mortality.

3. Early treatment of PDA significantly reduces BPD compared to later treatment.

\section{COULD CARDIAC ENZYMES AND THE CARINAL ANGLE MEASUREMENT BE USED AS INDICATORS OF HEMODYNAMICALLY SIGNIFICANT PATENT DUCTUS ARTERIOSUS?}

doi:10.1136/archdischild-2012-302724.0322

${ }^{1} \mathrm{E}$ Rahimov, ${ }^{2} \mathrm{Y}$ Ergul, ${ }^{3} \mathrm{Z}$ Ince, ${ }^{4} \mathrm{E}$ Yekeler, ${ }^{3} \mathrm{~S}$ Yildirim, ${ }^{5} \mathrm{~B}$ Omer, ${ }^{3} \mathrm{~A}$ Coban. ${ }^{1} \mathrm{C}$ Children's Health and Diseases; '2Pediatric Cardiology; ${ }^{3}$ Neonatology; ${ }^{4}$ Pediatric Radiology; ${ }^{5}$ Biochemistry, Istanbul University Istanbul Medical Faculty, Istanbul, Turkey

Aim In this blind prospective study, we aimed to examine the utility of cardiac enzymes and the carinal angle measurement in detecting hemodynamically significant patent ductus arteriosus (PDA), evaluating response to treatment and follow-up in preterm infants. Patients and Methods We evaluated 39 preterm infants $<32$ gestational weeks. Every patient had an echocardiogram (ECHO) taken on postnatal day 3 by a cardiologist blinded to clinical findings and laboratory results. ECHO was repeated when necessary. N-terminal pro-brain natriuretic peptide (NT-proBNP), cardiac troponin T $(\mathrm{c} \operatorname{Tn} \mathrm{T})$, creatine kinase $\mathrm{MB}$ isoenzyme (CK-MB) levels were measured on postnatal days 0,3 and 7 . The carinal angle on chest radiographs taken on the same days was measured. Those with PDA deemed hemodynamically significant based on ECHO were referred to as ehsPDA group. Those in this group who also had clinical signs were referred as sPDA subgroup.

Results The mean gestational age was 28.7 weeks; mean birth weight was $1120 \mathrm{~g}$. Initial ECHO showed PDA in 27 patients (69.2\%), 11 of which (40.7\%) had ehsPDA. Eight patients had sPDA When the ehsPDA and sPDA groups were compared to the rest, no statistically significant difference was found in terms of the carinal angle, NT-proBNP and cTnT. CK-MB levels were significantly low in ehsPDA and sPDA groups on postnatal days 3 and $7(p=0.017$, $\mathrm{p}=0.026$, respectively).

Conclusion NT-proBNP, cTnT, CK-MB and the carinal angle were not found useful in detection and follow-up of hemodynamically significant PDA.

323 ROLE OF AMINO TERMINAL PRO-BNP IN DIFFERENTIATING CARDIAC FROM RESPIRATORY PROBLEMS IN EGYPTIAN NEONATES PRESENTING WITH RESPIRATORY DISTRESS

doi:10.1136/archdischild-2012-302724.0323 